\title{
Three-dimensional phase retrieval in propagation-based phase-contrast imaging
}

\author{
A. Ruhlandt, M. Krenkel, M. Bartels, and T. Salditt* \\ Institut für Röntgenphysik, Georg-August-Universität Göttingen, Friedrich-Hund-Platz 1, Göttingen, Germany
}

(Received 12 August 2013; published 25 March 2014)

\begin{abstract}
We present a solution to the phase problem in near-field $\mathrm{x}$-ray (propagation) imaging. The three-dimensional complex-valued index of refraction is reconstructed from a set of projections recorded in the near-field (Fresnel) setting at a single detector distance. The solution is found by an iterative algorithm based only on the measured data and the three-dimensional tomographic (Helgason-Ludwig) consistency constraint without the need for further a priori knowledge or other restrictive assumptions.
\end{abstract}

DOI: 10.1103/PhysRevA.89.033847

PACS number(s): 42.30.Rx, 87.57.Q-, 87.59.-e

\section{INTRODUCTION}

$\mathrm{X}$-ray phase contrast is an emerging imaging technique which has seen considerable progress over the last 15 years [1-4]. Owing to the penetration power of hard $\mathrm{x}$ rays, the method is uniquely suitable for the investigation of bulk structures of unsliced, unstained, and (optically) nontransparent specimen. The long-standing limitation of x-ray imaging, that low-density and light-element tissues or samples were just "too tranparent" and nonabsorbing, has been overcome in revolutionary ways by the advent of phase-contrast techniques. Phase contrast can be achieved in different ways, notably by grating interferometry [5] or free propagation of partially coherent beams behind the object [6-8], encoding phase information into measurable intensities. While grating-based phase contrast is probably the method of choice for macroscopic medical imaging, propagation-based (near-field) imaging is better suited for high-resolution imaging, covering length scales from the centimeter range down to below $50 \mathrm{~nm}$. Aside from quantitative contrast and high resolution, the main advantage of $\mathrm{x}$ rays over other probes (visible light nanoscopy [9], electron microscopy) derives from its high penetration power. Therefore the most relevant applications will be in three dimensional (3D) structure analysis, enabled by computed tomography (CT) and the fact that image formation is sufficiently well described by line integrals through the tissue (projection approximation) [10,11].

The main challenge of propagation (or holographic) imaging is the phase-retrieval step based on intensity measurements in the detection plane. Several phase-retrieval methods have been developed, based for example on linearization of the contrast formation leading to simplified versions of the transport of intensity equation [12-14], or to an analytic form of the free-space contrast transfer functions (CTFs) [7,15-18]. Nevertheless, all present phase-retrieval techniques, whether based on Fourier filtering or iterative algorithms, require one or several additional assumptions like negligible absorption, slowly varying phase shifts, known relationships between phase shift and absorption, or known compact support of the object [19-21] to compensate the artifacts introduced by zeros in the oscillatory CTFs. So far, the only way to overcome these restrictions has been to use data sets with more than a single object to detector distance [22-24]. What has so far been missing is the capability to reconstruct objects with arbitrary

*Corresponding author: tsaldit@gwdg.de and unknown composition and structure from the measured intensity distribution in a single detection plane without restrictive additional assumptions. However, in the relevant case of tomography, single distance projections for different angles of the object are necessarily available. It is well known that these projections are not independent of each other [25,26] because the finite size of the sample imposes systematic correlations in particular for the low spatial frequencies of neighboring projections. This so-called Helgason-Ludwig consistency condition has been the starting point for reconstruction of incomplete data (missing wedge) in classical absorption-based CT [27]. The idea of the present work is to use this consistency as an inherent constraint to solve the phase problem in the holographic imaging regime, eliminating the need of additional $a$ priori knowledge. The idea is implemented by a nested phaseretrieval and tomographic-reconstruction scheme, which stabilizes the reconstruction of low spatial frequencies which have previously hampered reconstruction of single-image data, in particular for specimens where no additional constraints could be applied. More generally, consistency constraints relating several data sets of an object (projections, single exposures with overlap, images recorded at different photon energy) often warrant successful reconstruction. The recent development of ptychographic reconstruction techniques also forms an excellent example for this strategy [28-30].

In previous combinations of tomographic reconstruction and near-field phase retrieval [31-33], the process is separable into a first step of phase retrieval for each projection independently, followed by tomographic reconstruction, performed by filtered back projection (FBP), in a second step. The tomographic reconstruction is thus carried out on a set of projections for which the preceding phase retrieval is considered as final. In far-field coherent diffractive imaging, $3 \mathrm{D}$ phase retrieval by use of the $3 \mathrm{D}$ fast Fourier transform is easily implemented to the advantage of better convergence properties, but still necessitates a support constraint $[34,35]$. In contrast, in this work the representation of the object is strictly three dimensional and a coupled algorithm based on iterative phase retrieval and an algebraic tomographic reconstruction technique (ART) [36] is proposed, automatically exploiting the consistency condition. Based on simulated as well as on experimental data, we show that the combined (or coupled, nested) phase retrieval and tomography approach yields significantly enhanced reconstructions with considerably less input of a priori knowledge on the sample, even in the presence of noise. To demonstrate this, we present a specific algorithmic implementation, as given below. 


\section{PROPAGATION-BASED PHASE-CONTRAST TOMOGRAPHY}

The principle of phase-contrast tomography based on freespace propagation is sketched in Fig. 1(a). The object $\Omega$ is rotated around the $y$ axis, perpendicular to the propagation direction $z$ of a monochromatic plane wave $I(z)=I_{0} \exp (i k z)$ with wave number $k$. The interaction between object and wave is described by the complex index of refraction, which for $\mathrm{x}$ rays is commonly written as $n=1-\delta+i \beta$, where $\delta$ characterizes the phase shift and $\beta$ the absorption of the material. Note that for hard $\mathrm{x}$ rays around $10 \mathrm{keV}$ and soft tissue, $\delta$ is on the order of $10^{-6}$ and $\beta$ is up to three orders of magnitude smaller. The exit wave of an object rotated by an angle $\alpha$ in the $x y$ plane directly behind $(z=0)$ the object can be expressed in the projection approximation [2] as

$$
\begin{aligned}
\Phi(x, y)_{\alpha} & =I_{0} \exp \left(i k \int_{\Omega} n(x, y, z)_{\alpha} d z\right) \\
& \propto \exp \left[-i \mathcal{P}\left(\tilde{\delta_{\alpha}}\right)\right] \exp \left[-\mathcal{P}\left(\tilde{\beta_{\alpha}}\right)\right],
\end{aligned}
$$

with the projection operator $\mathcal{P}\left(\tilde{\delta}_{\alpha}\right)(x, y)=\int_{\Omega} \tilde{\delta}_{\alpha}(x, y, z) d z$ for $\tilde{\delta}_{\alpha}:=\delta_{\alpha} k$ and $\mathcal{P}\left(\tilde{\beta}_{\alpha}\right)$, respectively, which is in principle the two-dimensional Radon transform of $\tilde{\delta}$ and $\tilde{\beta}$. For the simulation below, the distance $d_{z}$ between sample and detector is chosen such that the Fresnel number $F:=a^{2} /\left(\lambda d_{z}\right) \approx 1$, corresponding to holographic contrast formation, where $a$ is a characteristic size of the object and $\lambda=2 \pi / k$ the wavelength. However, the main conclusions do not depend on the particular choice of $F$. Tomography can be well described in Fourier space via the projection-slice theorem [37], which identifies the Fourier transform of a projection with a radial slice through reciprocal space under the angle $\alpha$ corresponding to the projection. All slices share common values at the origin. For objects or reconstruction volumes of finite size $L$ (band limited), convolution with the object shape function broadens each Fourier slice to a thickness $d \propto 1 / L$, as sketched in Fig. 1(b), and depending on the edges may also introduce longrange tails, for example in the form of a sinc function for the simple case of a quadratic object. A mathematical formulation of the coupling between the projections in real space is given by the Helgason-Ludwig consistency condition [25-27]. This coupling of different projections is ideally suited to improve phase retrieval in propagation imaging, in particular for low spatial frequencies which are poorly transferred and otherwise only loosely constrained. In order to use this advantage, it is necessary to couple phase retrieval with tomographic reconstruction, i.e., to combine the two previously sequential steps.

\section{RECONSTRUCTION ALGORITHM}

As a reference, we use a sequential procedure that first reconstructs the phase of each detector image and afterwards combines all results into a $3 \mathrm{D}$ object. The phase retrieval is based on an iterative algorithm which cycles between detection plane and object plane, as sketched in Fig. 1(c). The procedure is similar to but more general than the well-known Gerchberg-Saxton (GS) algorithm [19,38] and is therefore denoted as a modified GS (MGS) scheme. Starting with the real-valued modulus of the holographic images $\left|\Psi_{0}\right|$ in the detection plane, all images are propagated back to the object plane using the inverse Fresnel propagator $\mathcal{D}_{-z_{d}}$. This kind of holographic reconstruction leads to severely perturbed reconstructions $\Phi^{\prime}$ due to the fact that the phase information is unknown. Since tomography requires the validity of the projection approximation, energy conservation and positivity of the electron density result in $|\Phi| \leqslant 1$ and $\arg (\Phi) \leqslant 0$, which is equivalent to $\delta \geqslant 0$ and $\beta \geqslant 0$. After applying these unrestrictive constraints, the $i$ th iterate $\Phi_{i}$ is propagated forwards to the detection plane, yielding $\Psi_{i}^{\prime}$. Finally, the amplitude constraint $\Psi_{i+1}=\Psi_{i}^{\prime}\left|\Psi_{0}\right| /\left|\Psi_{i}^{\prime}\right|$ changes the image amplitude to the measured values $\left|\Psi_{0}\right|$ while keeping the phase information. This procedure is then repeated several times and is called the outer loop in the following. Instead of imposing the restrictive condition $\beta=0$ valid only for a pure phase object or $\beta / \delta=$ const (single-material assumption), this approach imposes only conditions which are applicable to all objects. Note that the constraint $\beta \geqslant 0$ has been shown before to improve the quality of holographic reconstructions [39].

While convergence and fixed-point properties of similar iterative phase-retrieval algorithms have been well studied and have seen continuous adaptations and generalizations with (a)

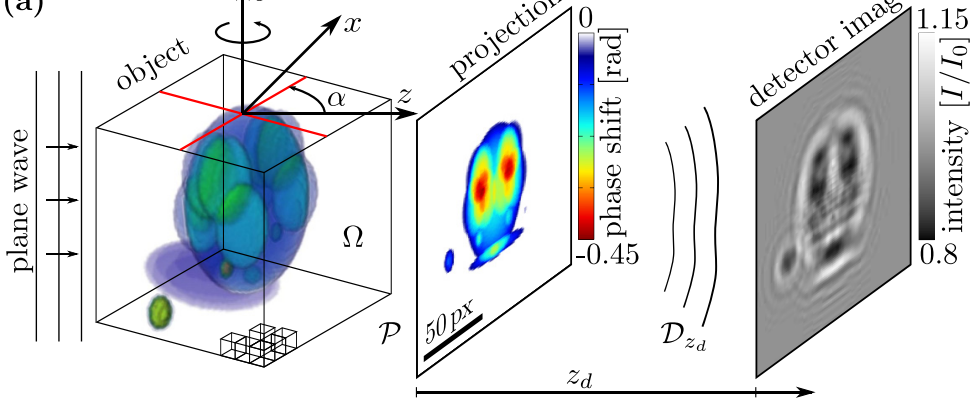

(b)

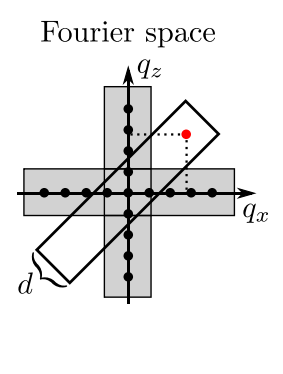

(c)

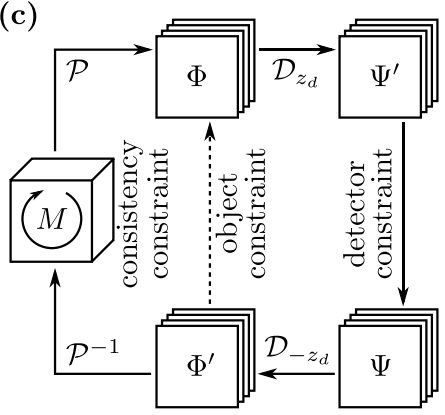

FIG. 1. (Color online) (a) Schematic of holographic phase-contrast imaging. A plane wave illuminates the object $\Omega$, leading to an exit wave in the $x y$ plane as given by the projected optical indices, followed by free-space propagation, resulting in holographic phase-contrast formation as recorded in the detection plane at distance $z_{d}$. (b) The finite cross section $L$ of the reconstruction volume leads to an effective width $d \propto 1 / L$ of the Fourier slices, characterizing correlated areas in reciprocal space. This width results in a coupling between different projections in particular at low spatial frequencies, providing a powerful constraint for phase retrieval. (c) Sketch of the iterative reconstruction algorithm devised to retrieve both the phase information and the three-dimensional distribution of the optical indices of the object. The Gerchberg-Saxton-like step explained in the text is represented by the dashed line. 
respect to the employed constraints, one decisive difference with respect to earlier work has to be stressed. Rather than being performed independently for each projection, the update $\Phi_{\alpha}^{\prime} \rightarrow \Phi_{\alpha}$ can be performed by an inner cycle of tomographic reconstruction followed by reprojection from the $3 \mathrm{D}$ volume to the exit plane corresponding to $\alpha$. This effectively imposes the consistency between different projections.

Combining the previously sequential steps of phase retrieval and tomography, in each outer loop the complex-valued $\Phi_{\alpha}^{\prime}$ are separated into a phase-shift part $\Phi_{\alpha, \tilde{\delta}}^{\prime}=-\left|\arg \left(\Phi_{\alpha}^{\prime}\right)\right|$ and an absorption part $\Phi_{\alpha, \tilde{\beta}}^{\prime}=-\ln \left[\min \left(\Phi_{\alpha}^{\prime}, 1\right)\right]$, applying the respective constraints ensuring $\delta \geqslant 0$ and $\beta \geqslant 0$. The threedimensional volumes $\tilde{\delta}(x, y, z)$ and $\tilde{\beta}(x, y, z)$ are reconstructed independently using a modified ART. Afterwards, the volumes are projected and combined into new $\Phi_{\alpha}$. We denote this nested iterative $3 \mathrm{D}$ reconstruction scheme which intrinsically obeys the consistency constraint as "iterative reprojection phase retrieval" (IRP). Stated otherwise, instead of a set of $2 \mathrm{D}$ projections, the reconstruction volume is strictly three dimensional throughout the entire reconstruction process. The exact ratios of outer phase retrieval and inner $3 \mathrm{D}$ reconstruction steps, however, can be chosen, i.e., optimized during the reconstruction, as discussed below.

\section{SIMULATIONS AND RESULTS}

To implement the tomographic reconstruction, we have devised an algorithm which can be considered as a modified ART. Compared to the FBP it has the advantage of introducing fewer high-frequency artifacts, which would inhibit the overall reconstruction [40]; see Appendix B The algorithm employs an envelope $M_{0}=\min \left[\mathcal{P}^{-1}\left(\Phi_{\alpha}^{\prime}\right)\right] \forall \alpha$ as initial guess for the $3 \mathrm{D}$ volume, where each voxel is set to the maximum value which it can possibly have, considering all $N$ projections (and analogously for $\tilde{\beta})$. The back-projection operator is defined as $\mathcal{P}_{\alpha}^{-1}: M(x, y, z)_{\alpha}=\Phi^{\prime}(x, y)_{\alpha}$. The next approximation $M_{i+1}$ to the $3 \mathrm{D}$ volume for either $\tilde{\beta}$ or $\tilde{\delta}$ is then calculated as follows:

$$
M_{i+1}=\frac{1}{N} \sum_{\alpha} M_{i} \mathcal{P}^{-1}\left(\frac{\Phi_{\alpha}^{\prime}}{\mathcal{P}\left(M_{i}\right)_{\alpha}}\right) .
$$

By this update step it is ensured that for each angle the projection of the volume fulfills the current projection guess $\Phi_{\alpha}^{\prime}$. By averaging over all $N$ projections, it is guaranteed that this algorithm converges to the least-squares solution for inconsistent projections [40]. This inner loop is repeated $\kappa$ times and afterwards the outer loop is continued, producing a new guess in the detection plane.

The quality of the reconstruction is monitored by an error metric according to

$$
\sigma^{2}:=\frac{1}{N n_{x} n_{y}} \sum_{\alpha, x, y}\left[\Psi^{\prime}(x, y)_{\alpha}-\Psi_{0}(x, y)_{\alpha}\right]^{2},
$$

comparing the modified $\Psi^{\prime}$ to the recorded detector images $\Psi_{0}$. Here, $n_{x} n_{y}$ denotes the number of detector pixels. Thus, $\sigma$ represents the average error per pixel in the detection plane, considering all $N$ projections. We have made the observation, that the quality of the tomographic reconstruction needs to be adapted to the phase-retrieval quality as a small number of iterations in the inner loop can limit the quality of the outer loop. One way to assure this is to double the number $\kappa_{i}$ of tomographic iterations when $\Delta \sigma:=\sigma_{i-1}-\sigma_{i}<\varepsilon$, starting with $\kappa_{0}=1$ (the first inner-loop iteration). A stopping criterion for the outer loop can thus be a maximum number of inner iterations $\kappa_{\max }$, as used in this work.

To test the validity and performance of the algorithm, we have used a phantom composed of $130^{3}$ cubic voxels, representing a superposition of several ellipsoids that differ in scale and value. Holographic intensity distributions were simulated for $N=90$ equidistant angles in the range $0^{\circ} \leqslant \alpha<$ $180^{\circ}$. One exemplary projection image and one holographic detector image are shown in Fig. 1(a). Figure 2 illustrates the advantages of IRP compared to the MGS scheme and thereby the quality of the consistency constraint. Figure 2(a) shows a slice through the phase volume and a histogram of the phantom depicting the frequency of the different phase-shift values. For a pure phase object $(\beta=0)$, both the sequential reconstruction approach (MGS) shown in Fig. 2(b) and the coupled approach (IRP) shown in Fig. 2(c) yield quite good $3 \mathrm{D}$ phase reconstructions. However, the latter shows fewer image artifacts. As quantified by the histograms, the IRP reconstruction allows for a better separation of the different phase-shift values. In particular, the lowest value of $\tilde{\delta}$ can be distinguished from the noisy underground only in the histogram of the IRP reconstruction. For more realistic cases, $\beta$ is always at least slightly larger than zero. Simulations show that already small values $\beta / \delta \approx 0.003$, in the range expected for soft biological tissues, lead to a decrease of the reconstruction quality in the separate reconstruction scheme (MGS) and to systematic errors by wrongfully forcing $|\Phi|=1$. If the ratio $\beta / \delta$ is known and the value is used as an additional constraint, applicable to samples of varying density but identical stochiometry (single material), the conventional approach is also able to reconstruct the volume sufficiently well [24]. However, in most cases, $\beta / \delta$ ratios are not known or the object consists of more than one material, leading to spatially varying $\beta / \delta$ ratios. For a simulated object with different $\beta / \delta$ ratios for each ellipsoid (ranging from 0.01 to 0.075 ), the conventional approach (MGS) shows significant artifacts, especially for lower spatial frequencies; see, for example, the large hole in the center of the reconstructed phantom as shown in Fig. 2(d). IRP reconstruction, on the other hand, shows only slight deviations, as shown in Fig. 2(e), and is still capable of separating the materials based on the better fidelity in voxel values (histogram). Even completely uncorrelated $\beta / \delta$ distributions can be reconstructed, demonstrating the power of the 3D consistency constraint (see the Appendix C for further details). Finally, IRP converges much faster with respect to the number of MGS loops required ( $\leqslant 400$ vs 10000 ), but it should be mentioned that the tomography step in each iteration is computationally very expensive, at least if ART is used.

\section{RECONSTRUCTION OF NOISY AND EXPERIMENTAL DATA}

Figure 3 illustrates the influence of noise on the reconstruction. For the case shown, Gaussian noise with a standard deviation of $\sigma=0.01$ was added to every simulated detector intensity image for the case of a pure phase object [ $\beta=0$, Fig. 3(a)]. The IRP reconstruction shown in Fig. 3(c) 


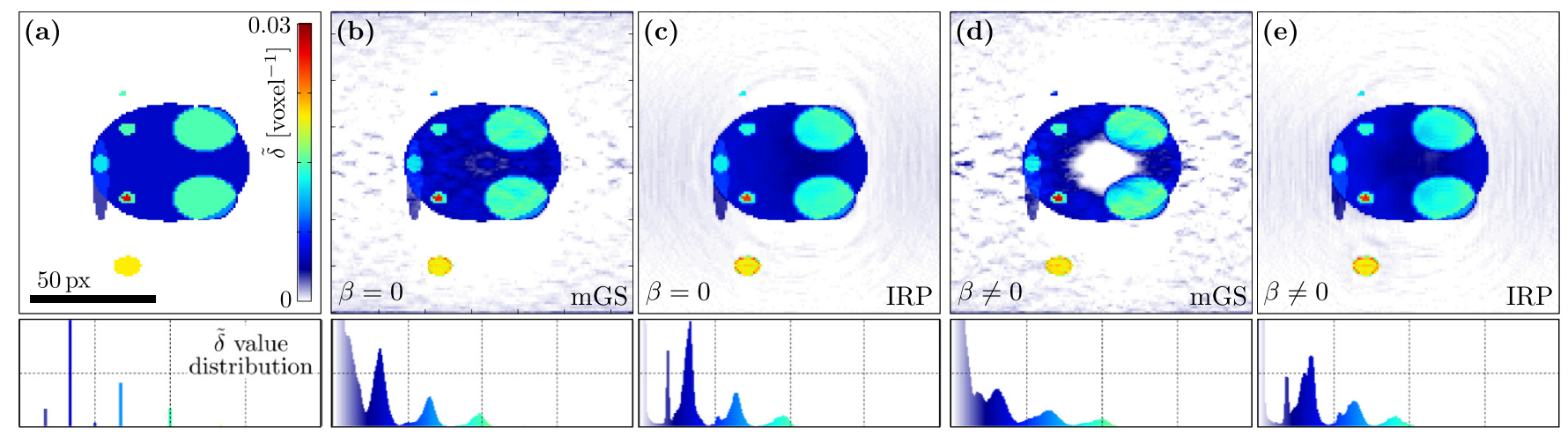

FIG. 2. (Color online) Reconstruction quality for the standard approach of separated phase and tomographic reconstruction compared to the coupled reconstruction according to IRP, each for the case of a pure phase object and a mixed object. (a) Phase shift $\tilde{\delta}$ of the reference phantom. (b) Reconstructed phase shift of a pure phase object $\beta=0$ using the MGS algorithm (20 000 GS loops, followed by 128 ART iterations). The pure phase constraint has been enforced in the reconstruction. (c) Same as in (b) but with IRP reconstruction $\left(\kappa_{\max }=128\right.$ ART iterations), and without using the fact that the phantom was a phase object. (d) Reconstructed phase shift of an object with fixed $\beta / \delta=0.02$ coupling, using the modified GS algorithm (20000 GS loops, followed by 128 ART iterations). As in (b) the coupling value was enforced. (e) As in (d) but with IRP reconstruction ( $\kappa_{\max }=128$ ART iterations), and without using any preknown coupling values. Slices of reconstructed volumes in the $y z$ plane (top row) are shown to illustrate the difference in data quality, along with the histograms of the $\tilde{\delta}$ values for the entire volume (bottom row). The scale bar applies to the entire row, the color bars to all images and the histograms. The frequency of occurrence in the histograms is shown in linear scale from 0 to 5000 counts.

substantially outperforms the result obtained by the conventional sequential scheme (MGS), depicted in (b). To test the IRP algorithm on experimental data, we finally applied the reconstruction to a phase-contrast data set of Deinococcus radiodurans bacterial cells, recorded by tomographic x-ray propagation microscopy [21], i.e., tomography in a cone beam with geometric magnification and phase-contrast formation by free-space propagation. The freeze-dried bacteria were positioned $8 \mathrm{~mm}$ behind a quasipoint source formed by an $\mathrm{X}$-ray waveguide, resulting in an effective pixel size of $83 \mathrm{~nm}$. A typical holographic intensity image (experimental data) is shown in Fig. 3(d). Previously the data were treated in the sequential mode, first using phase retrieval based on a mod- ified hybrid-input-output algorithm (MHIO) with a support constraint, followed by FBP of all phased projections. A major challenge of previous phase retrieval was the automatic support determination for all projection angles [21]. A slice through the reconstruction volume is shown in Fig. 3(e) for the original sequential reconstruction (MHIO) and in (f) for IRP, showing an excellent quality with a more evenly distributed signal than before without any additional support information.

\section{SUMMARY}

In summary, we have shown that by coupling the phase retrieval and tomographic reconstruction, the phase problem
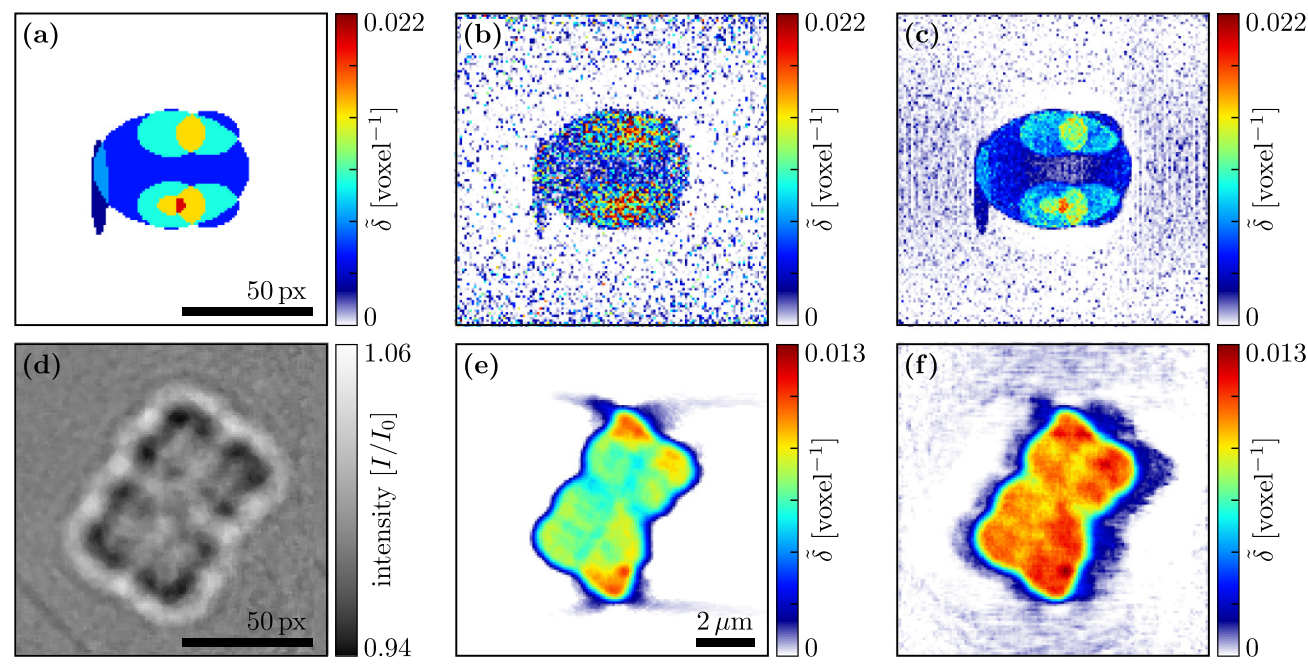

FIG. 3. (Color online) Influence of Gaussian noise and reconstruction of experimental data. (a) $x y$ slice of the undisturbed pure phase object. (b) $x y$ slice of the MGS reconstruction from noisy pure phase object images ( $\sigma=0.01,20000$ loops, followed by 128 ART iterations). (c) $x y$ slice of the IRP reconstruction from the same detector images as in (a) ( $\kappa_{\max }=128$ ART iterations). (d) Typical holographic diffraction pattern (detector plane) of a bacterial cell [21]. (e) Slice though the modified HIO reconstruction as described in [21]. (f) Slice through the IRP reconstruction from the experimental data, based on 83 projections. 
in full field (Fresnel) x-ray propagation imaging can be solved beyond the current restrictions. In particular, no assumptions either on the phase-shifting and absorption properties of the object or on the support are necessary. Instead, redundancy of the data in view of the unknowns is derived from the tomographic consistency condition, i.e., the correlations in different projections which are inherent in finite-size volumes, but had not been exploited for near-field phase retrieval before. While the general principle of the nested or coupled reconstruction holds for many different implementations of the phase retrieval and tomography, the specific algorithmic example IRP exhibits a particularly high performance; however, at the cost of significant computational effort. It can be expected that many corrections to imperfect data as well as regularization strategies can be accommodated in this scheme. A first investigation with noisy data proved the robustness of the IRP algorithm. Towards faster reconstruction, in particular needed for images with larger pixel number, one should consider a coupled phase retrieval and tomography scheme which replaces ART by FBP. For experimental data, good reconstructions have been achieved without additional support information or assumptions about the sample. With the presented approach, $\mathrm{x}$-ray phase-contrast propagation imaging is now equipped with a means to treat images recorded at a single detector distance, and for the most general case of objects.

An example program performing the IRP reconstruction is included as Supplemental Material [41].

\section{ACKNOWLEDGMENTS}

We thank Markus Osterhoff for helpful discussions and in particular for his advice on numerical issues, and acknowledge support by the Collaborative Research Center 755 Nanoscale Photonic Imaging of the German science foundation (DFG).

\section{APPENDIX A: PHANTOM DEFINITION}

The phantom used in this work consists of $130 \times 130 \times 130$ voxels, representing a sum of 17 ellipsoids with different sizes and values, each defined by the formula

$$
\tilde{\delta}(x, y, z)= \begin{cases}v_{i} & \text { if }\left(\frac{x-c_{x}}{a}\right)^{2}+\left(\frac{y-c_{y}}{b}\right)^{2}+\left(z-c_{z}\right)^{2}<r^{2} \\ 0 & \text { else. }\end{cases}
$$

TABLE I. Parameters of all ellipsoids that make up the phantom. The unit vx indicates the size of one voxel.

\begin{tabular}{lcrcclll}
\hline \hline Ellipsoid & $c_{x}(\mathrm{vx})$ & $c_{y}(\mathrm{vx})$ & $c_{z}(\mathrm{vx})$ & $r(\mathrm{vx})$ & $a(\mathrm{vx})$ & $b(\mathrm{vx})$ & $v_{i}(\mathrm{vx})$ \\
\hline 1 & 0 & 0 & 0 & 34.18 & 0.55 & 0.75 & 0.005 \\
2 & -8.92 & -14.86 & 0 & 14.86 & 0.5 & 0.7 & 0.005 \\
3 & -8.92 & 14.86 & 0 & 14.86 & 0.5 & 0.7 & 0.005 \\
4 & 8.92 & -14.86 & 0 & 14.86 & 0.5 & 0.7 & 0.005 \\
5 & 8.92 & 14.86 & 0 & 14.86 & 0.5 & 0.7 & 0.005 \\
6 & 8.92 & 14.86 & 0 & 7.43 & 0.5 & 0.7 & 0.005 \\
7 & -2.97 & -14.86 & 14.86 & 14.86 & 0.5 & 0.7 & 0.005 \\
8 & -2.97 & 14.86 & 14.86 & 14.86 & 0.5 & 0.7 & 0.005 \\
9 & 2.97 & -14.86 & 14.86 & 14.86 & 0.5 & 0.7 & 0.005 \\
10 & 2.97 & 14.86 & 14.86 & 14.86 & 0.5 & 0.7 & 0.005 \\
11 & -0.74 & -14.86 & -18.28 & 4.46 & 0.5 & 0.7 & 0.01 \\
12 & -0.59 & 14.86 & -18.58 & 2.97 & 0.5 & 0.7 & 0.012 \\
13 & 0.45 & -29.72 & -20.36 & 1.49 & 0.5 & 0.7 & 0.015 \\
14 & 0.30 & 44.58 & -18.43 & 5.94 & 0.5 & 0.7 & 0.02 \\
15 & -0.74 & 14.86 & -18.28 & 4.46 & 0.5 & 0.7 & 0.01 \\
16 & 14.86 & 7.43 & -29.72 & 3.72 & 8 & 5.5 & 0.0025 \\
17 & 0 & 0.00 & -29.72 & 3.72 & 1 & 1 & 0.005 \\
\hline \hline
\end{tabular}

The parameters $c_{x}, c_{y}, c_{z}, a, b$, and $r$ are listed in Table I. Due to the overlap of some ellipsoids, the phantom shows nine different values from 0.005 to 0.027 .

\section{APPENDIX B: COMPARISON OF TOMOGRAPHIC RECONSTRUCTIONS}

The deviations of the reconstructed volumes $M_{\text {rec }}$ from the ideal phantom $M_{\mathrm{ph}}$ are measured by the root mean square deviation

$$
\sigma:=\sqrt{\frac{1}{N} \sum_{i=1}^{N}\left(M_{\mathrm{ph}, i}-M_{\mathrm{rec}, i}\right)^{2}}
$$

where $N$ denotes the number of voxels (here $N=130^{3}$ ). The phantom was projected to 90 equidistant angles fom 0 to $180^{\circ}$ and reconstructed with the presented algebraic reconstruction technique implementation and the common filtered back projection. The results are compared in Fig. 4 for a slice perpendicular to the rotation axis. The ART shows less high-frequency noise, leading to $\sigma_{\mathrm{ART}}=8.19 \times 10^{-5}$ after 128 iterations. The FBP yields $\sigma_{\mathrm{FBP}}=2.88 \times 10^{-4}$. Even if
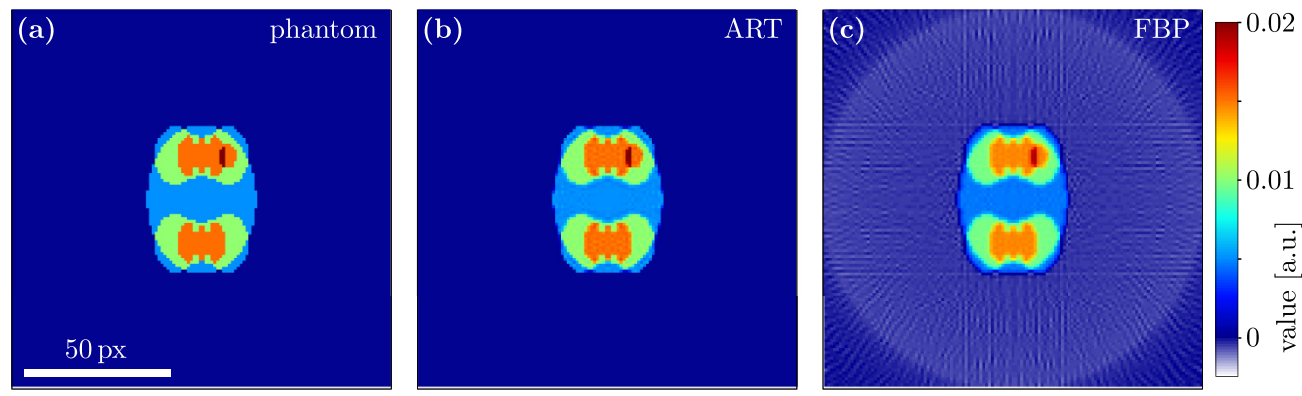

FIG. 4. (Color online) Comparison of tomographic reconstruction (no phase retrieval) by ART and FBP. (a) shows a slice through the phantom perpendicular to the axis of rotation. The volume was projected onto 90 equidistant angles fom 0 to $80^{\circ}$ and reconstructed using (b) ART (128 iterations) and (c) FBP. 

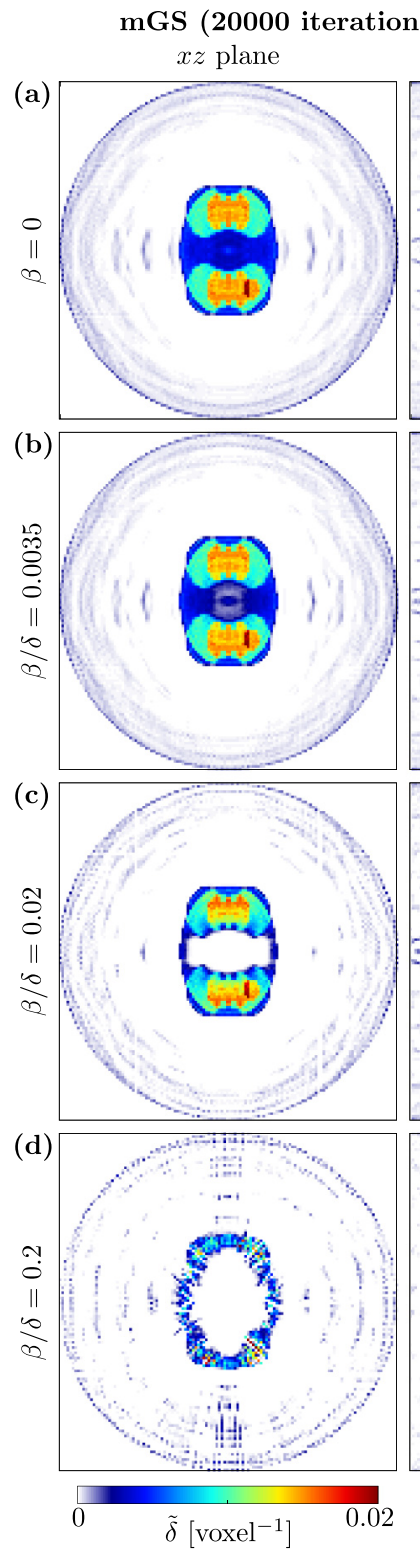
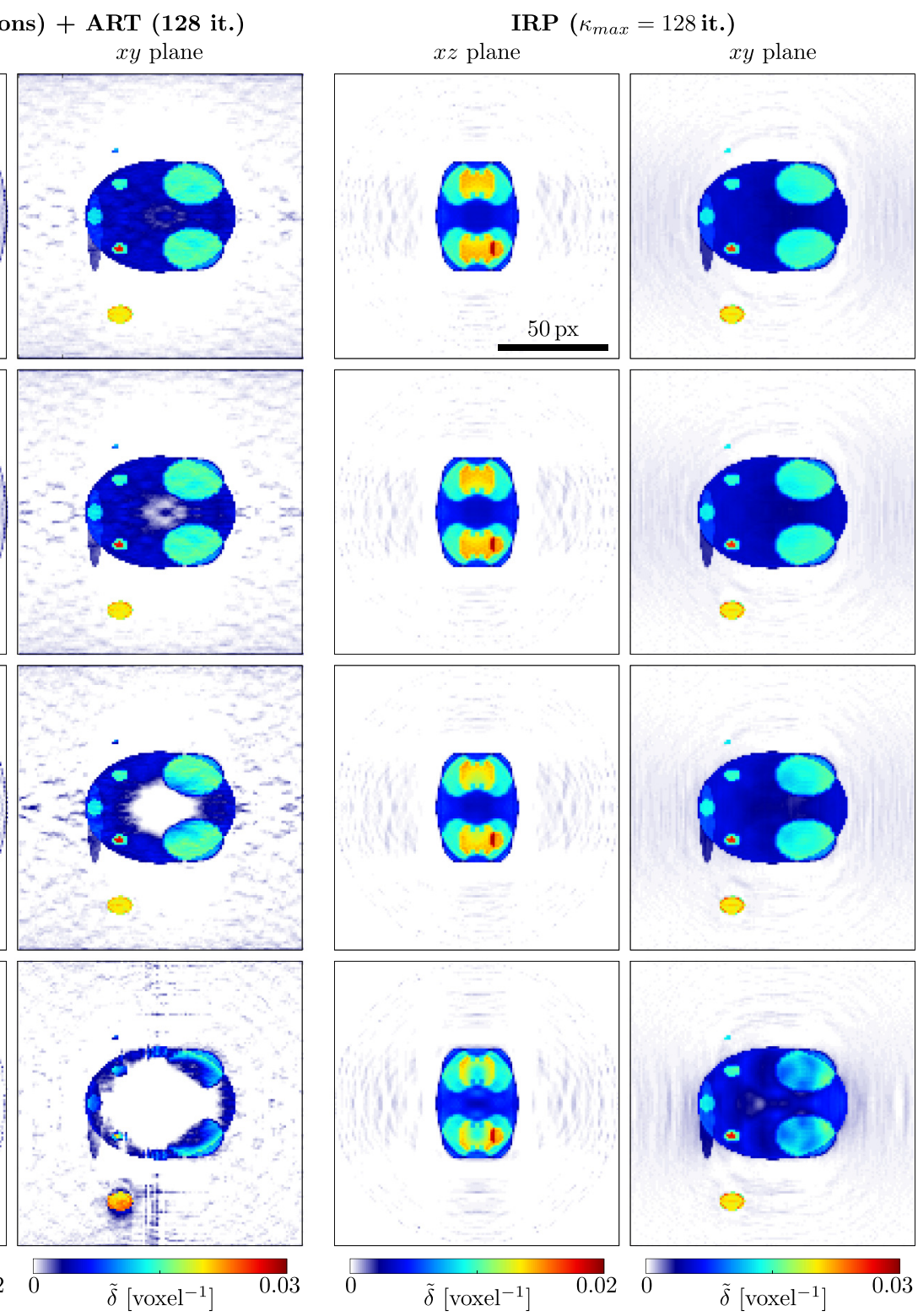

FIG. 5. (Color online) Influence of the $\beta / \delta$ ratio (rows) on the reconstruction results for the MGS and IRP algorithms (columns).

the nonphysical negative values are set to zero, the standard deviation is $\sigma_{\mathrm{FBP}>0}=1.63 \times 10^{-4}$.

\section{APPENDIX C: OBJECTS WITH MIXED PHASE AND AMPLITUDE CONTRAST}

This appendix presents more simulation results on objects with mixed phase and amplitude contrast. Several established phase-retrieval algorithms perform very well for pure phase objects. However, even for hard $\mathrm{x}$ rays and soft tissues a small contribution of the absorption is expected and can spoil proper reconstruction, highlighting the importance of phase retrieval for more general mixed objects. The influence of a small $\beta / \delta$ ratio on the reconstruction results is illustrated in Fig. 5. The results for the conventional sequential approach (MGS) is compared to IRP for cases $\beta / \delta=0$ up to $\beta / \delta=0.2$. In all cases, the ratio was unknown to the algorithms and hence not used as a constraint. In both algorithms, only the "mild" constraints $|\Phi| \leqslant 1$ and $\arg (\Phi) \leqslant 0$ were used. The $\sigma$ values for the different results shown in Fig. 5 are presented in Table II. It can be seen that the consistency condition in IRP leads to better results in all cases.

TABLE II. Standard deviation $\sigma \times 10^{3}$ for different reconstruction methods (rows) and different $\beta / \delta$ ratios (columns). The ART was stopped after 128 iterations, IRP was carried out until the inner loop reached 128 iterations.

\begin{tabular}{lcccc}
\hline \hline$\beta / \delta$ ratio & 0.2 & 0.02 & 0.0035 & 0 \\
\hline MGS and FBP & 1.18 & 0.59 & 0.40 & 0.37 \\
MGS and ART & 1.12 & 0.53 & 0.38 & 0.36 \\
IRP & 0.34 & 0.23 & 0.21 & 0.21 \\
\hline
\end{tabular}



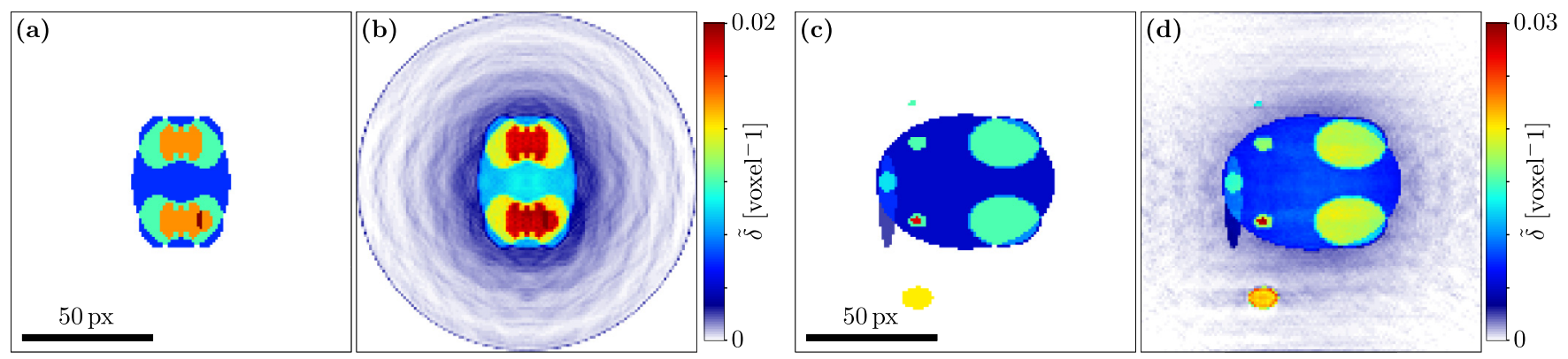

FIG. 6. (Color online) Influence of neglected weak absorption on the reconstruction. (a) Phantom $x z$ plane, perpendicular to the axis of rotation. (b) Reconstructed $x y$ plane using 20000 iterations of MGS with the constraint $|\Phi|=1$, followed by 128 iterations of ART. (c) Phantom $x y$ plane. (d) Reconstructed $x y$ plane.

Next, we address the question of whether the reconstruction quality can be improved by imposing the constraint of a pure phase object (i.e., forcing the amplitude to 1), if the object is weakly absorbing. In other words we check the validity of neglecting absoprtion for the purpose of stabilizing phase retrieval. Figure 6 shows reconstructions for the case of $\beta / \delta=0.02$, resulting in a maximum absorbtion of $1.42 \%$ and a mean absorption of $0.33 \% .90$ angles were simulated and reconstructed using MGS (20000 iterations) with the additional condition $|\Phi|=1$, i.e., no absorption. The threedimensional object was reconstructed using ART with 128 iterations. The results show much fewer distortions in the object shape and features [compare to Fig. 5(c) MGS] but a significant error in the values. Please note that this effect cannot be corrected for by a constant offset. The comparison with the phantom yields $\sigma=6.76 \times 10^{-4}$, which is inferior to all reconstructions shown in Table II. This shows that neglecting absorption even for very weakly absorbing objects yields inconsistent phase distributions.

Finally, we compare the reconstruction results for completely independent $\beta / \delta$ ratios. Figure 7 shows the results for $\tilde{\delta}$ and $\tilde{\beta}$ for a simulation with both the original phantom for $\tilde{\delta}$ and an independent absorption phantom. For the latter, the original phantom was rotated, enlarged, and set to lower values. Note that this includes the unphysical case of absorption without a phase shift. 180 equidistant angles from 0 to $180^{\circ}$ were simulated and reconstructed using MGS (14 000 iterations) and IRP (up to 128 inner ART loops). $\tilde{\delta}$ can be reconstructed to a nearly quantitative level using IRP and even the contour of the much weaker $\tilde{\beta}$ part can be retrieved. In contrast, the MGS reconstruction leads to heavily distorted volumes.
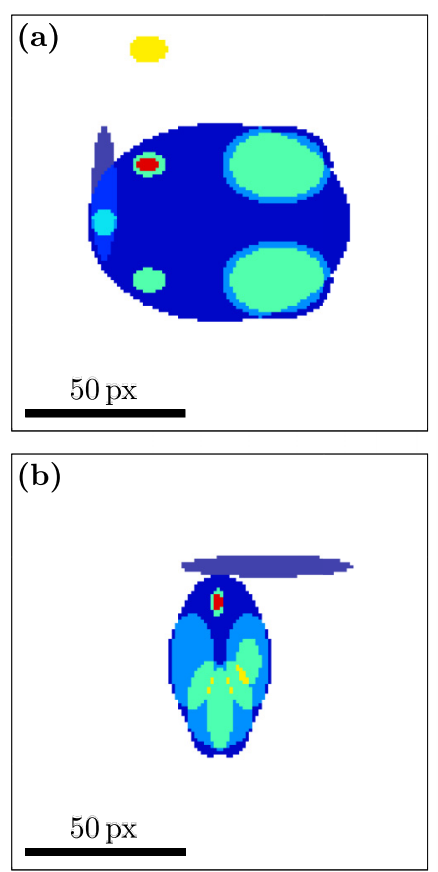
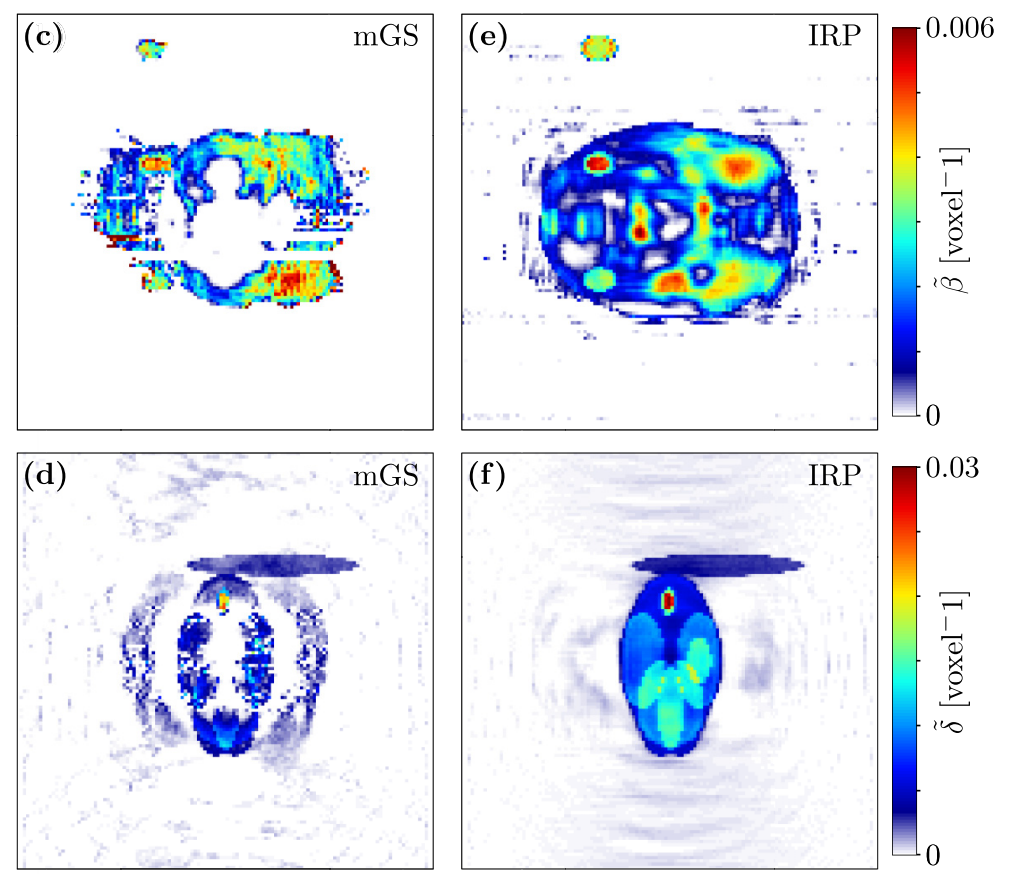

FIG. 7. (Color online) Reconstruction of completely independent $\beta / \delta$ values. The top row shows the $\tilde{\beta}$ part, the bottom row the $\tilde{\delta}$ part of the same volume. (a),(b) Phantom $x y$ plane. (c),(d) Same plane, MGS results. (e),(f) Same plane, IRP results. 
[1] K. A. Nugent, T. E. Gureyev, D. F. Cookson, D. Paganin, and Z. Barnea, Phys. Rev. Lett. 77, 2961 (1996).

[2] D. Paganin, Coherent X-Ray Optics (Oxford University Press, New York, 2006).

[3] G. J. Williams, H. M. Quiney, B. B. Dhal, C. Q. Tran, K. A. Nugent, A. G. Peele, D. Paterson, and M. D. de Jonge, Phys. Rev. Lett. 97, 025506 (2006).

[4] M. Dierolf et al., Nature (London) 467, 436 (2010).

[5] F. Pfeiffer, C. Kottler, O. Bunk, and C. David, Phys. Rev. Lett. 98, 108105 (2007).

[6] S. Wilkins et al., Nature (London) 384, 355 (1996).

[7] P. Cloetens et al., Appl. Phys. Lett. 75, 2912 (1999).

[8] D. Paganin and K. A. Nugent, Phys. Rev. Lett. 80, 2586 (1998).

[9] S. W. Hell and J. Wichmann, Opt. Lett. 19, 780 (1994).

[10] S. C. Mayo et al., Opt. Express 11, 2289 (2003).

[11] C. Olendrowitz et al., Phys. Med. Biol. 57, 5309 (2012).

[12] D. Paganin et al., J. Microsc. 206, 33 (2002).

[13] A. Groso et al., Opt. Express 14, 8103 (2006).

[14] A. V. Bronnikov, Opt. Commun. 171, 239 (1999).

[15] L. Turner et al., Opt. Express 12, 2960 (2004).

[16] T. Gureyev et al., Appl. Opt. 43, 2418 (2004).

[17] M. Langer et al., Opt. Lett. 37, 2151 (2012).

[18] R. Hofmann, J. Moosmann, and T. Baumbach, Opt. Express 19, 25881 (2011).

[19] R. W. Gerchberg and W. O. Saxton, Optik 35, 237 (1972).

[20] K. Giewekemeyer, S. P. Krüger, S. Kalbfleisch, M. Bartels, C. Beta, and T. Salditt, Phys. Rev. A 83, 023804 (2011).

[21] M. Bartels et al., Opt. Nanoscopy 1, 10 (2012).

[22] L. J. Allen and M. P. Oxley, Opt. Commun. 199, 65 (2001).
[23] H. You-Li et al., Chin. Phys. B 21, 104202 (2012).

[24] M. Krenkel et al., Opt. Express 21, 2220 (2013).

[25] S. Helgason, Acta Math. 113, 153 (1965).

[26] D. Ludwig, Commun. Pure Appl. Math. 19, 49 (1966).

[27] H. Kudo and T. Saito, J. Opt. Soc. Am. A 8, 1148 (1991).

[28] J. M. Rodenburg, A. C. Hurst, A. G. Cullis, B. R. Dobson, F. Pfeiffer, O. Bunk, C. David, K. Jefimovs, and I. Johnson, Phys. Rev. Lett. 98, 034801 (2007).

[29] P. Thibault et al., Science 321, 379 (2008).

[30] A. J. D'Alfonso, A. J. Morgan, A. W. C. Yan, P. Wang, H. Sawada, A. I. Kirkland, and L. J. Allen, Phys. Rev. B 89, 064101 (2014).

[31] A. V. Bronnikov, J. Opt. Soc. Am. A 19, 472 (2002).

[32] T. E. Gureyev et al., Appl. Phys. Lett. 89, 034102 (2006).

[33] R. Mokso et al., Appl. Phys. Lett. 90, 144104 (2007).

[34] S. Marchesini et al., Opt. Express 11, 2344 (2003).

[35] A. Barty et al., Phys. Rev. Lett. 101, 055501 (2008).

[36] R. Gordon et al., J. Theor. Biol. 29, 471 (1970).

[37] A. C. Kak and M. Slaney, Principles of Computerized Tomographic Imaging (IEEE Press, New York, 1988).

[38] J. R. Fienup, Opt. Lett. 3, 27 (1978).

[39] T. Latychevskaia and H.-W. Fink, Phys. Rev. Lett. 98, 233901 (2007).

[40] C. L. Byrne, Applied Iterative Methods (A. K. Peters, Wellesley, 2008).

[41] See Supplemental Material at http://link.aps.org/supplemental/ 10.1103/PhysRevA.89.033847 for an example program performing the IRP reconstruction. 